\title{
Correlation between kidney transplantation and colorectal cancer in hemodialysis patients: A nationwide, retrospective, population-based cohort study
}

\author{
Han-En Wang ${ }^{1}$, Yu-Chan Liao ${ }^{2}$, Je-Ming Hu ${ }^{3,4}$, Wen-Chih Wu' ${ }^{5}$, Wan-Yun Chou ${ }^{6}$, Yong-Chen Chen ${ }^{7,8}$,
} Yu-Ching Chou ${ }^{2}$, Chi-Feng Hung ${ }^{7,8}$, Yu-Feng Tian ${ }^{9,10}$, San-Lin You ${ }^{7,8}$ and Chien-An Sun ${ }^{8,11^{*}}$ (D)

\begin{abstract}
Background: Kidney transplantation (KT) correlates with an increased risk of developing several malignancies; however, the risk of colorectal cancer (CRC) after KT remains debatable and has been marginally explored. Hence, in this nationwide, retrospective, population-based cohort study, we aimed to examine the correlation between KT and CRC in a large-scale population-based Chinese cohort.

Methods: We identified a total of 3739 regular hemodialysis patients undergoing KT (exposed cohort) and 42,324 hemodialysis patients not undergoing KT (non-exposed cohort) between 2000 and 2008 from Taiwan's National Health Insurance Research Database (NHIRD). Both cohorts were followed up from January 1, 2000, to the date of CRC diagnosis, death, or the end of 2013. Using Kaplan-Meier method, we measured the cumulative incidence of CRC in each cohort. Furthermore, Cox proportional hazards models were used to compute hazards ratios (HRs) and 95\% confidence intervals (Cls) to estimate the correlation between KT and CRC in hemodialysis patients.

Results: The Kaplan-Meier analysis revealed that the cumulative incidence of CRC was significantly higher in the exposed cohort than in the non-exposed cohort (log-rank test, $P<0.001)$. After adjusting for potential confounders, the exposed cohort exhibited a significantly increased risk of CRC compared with the non-exposed cohort (adjusted $\mathrm{HR}, 1.34 ; 95 \% \mathrm{Cl}, 1.11-1.62)$.
\end{abstract}

Conclusions: Hemodialysis patients undergoing KT have a significantly higher risk of CRC than those not undergoing KT. Cancer should continue to be a primary focus of prevention during KT.

Keywords: Colorectal cancer, Hemodialysis, Kidney transplantation, Retrospective cohort study

\section{Background}

Kidney transplantation (KT) correlates with an increased risk of developing several malignancies. The overall incidence of cancer in kidney transplant recipients is estimated to be $1.9-18 \%$ [1-5]. Established evidence from registry data and observational studies have reported a 2.5 - to 3 -fold increase in the overall risk of cancer among kidney transplant recipients $[1,2]$. Furthermore,

\footnotetext{
* Correspondence: 040866@mail.fju.edu.tw

${ }^{8}$ Big Data Research Center, College of Medicine, Fu-Jen Catholic University,

New Taipei City, Taiwan

${ }^{11}$ Department of Public Health, College of Medicine, Fu-Jen Catholic

University, New Taipei City, Taiwan

Full list of author information is available at the end of the article
}

the risk exponentially increases with virus-associated neoplasms such as human papillomavirus-related urogenital cancers, human herpesvirus 8-associated Kaposi's sarcoma, and Epstein-Barr virus-related post-transplant lymphoproliferative disease, with an excess risk of, at least, 5-30 times higher than that in age- and sex-matched general population [4]. Perhaps, immunosuppression following transplantation is a critical factor in the augmented incidence of malignancy [6, 7]. The risk of carcinoma increases with cumulative and prolonged use of immunosuppressant agents [6, 7]. Advancements in surgical techniques and immunosuppressive therapies have led to enhanced survival rates of patients and grafts; thus, post-transplantation 
cancer development could be a critical cause of morbidity and mortality in these patients in the future.

Globally, colorectal cancer (CRC) is a leading cause of morbidity and mortality, accounting for $>9 \%$ of all cancer incidences. Reportedly, CRC is the third leading cancer worldwide and the fourth leading cause of cancerrelated mortality [8]. Most studies have reported an elevated risk of CRC among solid organ transplant recipients relative to the general population, with standardized incidence ratios (SIRs) ranging from no correlation to a 4.5-fold increase [1-5]; while a meta-analysis reported an overall SIR estimate of 1.69 [9]. Nevertheless, the risk of CRC after KT remains debatable and has been marginally explored. In addition, CRC has never been reported to be caused by a viral infection, and CRC risk is not high among human immunodeficiency virus-infected individuals who are also immunosuppressed, [9] increasing the likelihood of a different underlying biological mechanism. In Taiwan, end-stage renal disease (ESRD) causes substantial medical and economic burden [10]. Reportedly, KT is usually performed for ESRD and is the ideal alternative to dialysis [10]. Furthermore, CRC is a common malignant tumor in Taiwan, with $>15,410$ new cases diagnosed in 2013 and an incidence rate of 44.32 per 100,000 [11, 12]. Hence, this nationwide cohort study aimed to assess the CRC risk in KT recipients by reviewing Taiwan's National Health Insurance Research Database (NHIRD).

\section{Methods}

\section{Data source}

In this study, we retrieved data from the medical claim database of Taiwan's NHIRD-an extensive database provided by a single-payer, universal, compulsory health care system, National Health Insurance (NHI), for nearly all 23.7 million residents of Taiwan. To date, the NHIRD has been comprehensively used for high-quality epidemiological studies $[13,14]$ and information on diagnoses, prescriptions, and hospitalizations have been shown to be of good validity $[15,16]$. In the present study, we obtained data from the Longitudinal Health Insurance Database (LHID 2000), a subset of NHIRD that comprises historical ambulatory and inpatient care data of 1 million randomly sampled beneficiaries enrolled in the NHI system in 2000. Moreover, the LHID 2000 database has facilitated access to the medical service use history of patients under investigation. Notably, no marked differences were observed in terms of the distributions of age, sex, and health care costs between individuals in the LHID and NHIRD $[13,14]$.

\section{Participants}

In this nationwide, retrospective, population-based cohort study, we enrolled patients with a primary diagnosis of ESRD undergoing hemodialysis between January 1,
2000, and December 31, 2008, as per the LHID. This duration was defined as the exposure period to determine hemodialysis patients who received KT. Figure 1 summarizes the subject selection process. First, we identified patients diagnosed with ESRD [International Classification of Disease, Revision 9, Clinical Modification (ICD-9-CM) code: 585] undergoing hemodialysis (ICD9-CM code: 39.95) and received KT (ICD-9-CM codes: V42.0, 996.81, and V58.69) as the exposed cohort $(n=$ 1375). Comparatively, patients with ESRD who were undergoing hemodialysis but not receiving $\mathrm{KT}$ were identified as the non-exposed cohort $(n=24,703)$. Patients were excluded if they were younger than 30 years of age, had incomplete demographic data, or were ever diagnosed with CRC prior to the beginning of follow-up (January 1, 2000). Overall, a total of 3739 hemodialytic patients receiving KT (exposed group) and 42,324 not receiving KT (non-exposed group) were included in data analyses.

\section{Ascertainment of CRC}

The primary outcome was the primary diagnosis of CRC (ICD-9-CM codes: 153, 153.0, 153.1, 153.2, 153.3, 153.6, 153.7, 153.8, 153.9154, 154.0, 154.1, 154.2, 154.3, 154.8, and 159.0). In this study, CRC diagnosis was defined according to the Registry for Catastrophic Illness Patient Database (RCIPD), a subpart of the NHIRD. The diagnosis of CRC needs histologic confirmation to be reported in the RCIPD. We followed both cohorts from January 1,2000 , to the date of CRC diagnosis, death (indicated by withdrawal from the NHI), or the end of 2013, whichever occurred first.

\section{Potential confounders and comorbidities}

We noted the covariates that were the potential confounders in the association between $\mathrm{KT}$ and $\mathrm{CRC}$. The potential confounders considered in this study included age, sex, and comorbidities, [17] including chronic obstructive pulmonary disease (ICD-9-CM codes: 490, 491, 492, 493, 494, 495, and 496), diabetes mellitus (ICD-9-CM code: 250), coronary artery disease (ICD-9-CM codes: 410, 411, 412, 413, and 414), hypertension (ICD-9-CM codes: 401, 402, 403, 404, and 405), alcohol-related conditions (ICD-9-CM codes: alcoholic liver disease, 571.0, 571.1, 571.2, and 571.3 and alcohol dependence, 303), hypercholesterolemia (ICD-9-CM codes: 272.0, 272.1, 272.2, and 272.4), peptic ulcer (ICD-9-CM codes: 531, 532, and 533), liver cirrhosis and chronic hepatitis (ICD-9-CM code: 571), and inflammatory bowel disease (ICD-9-CM codes: 555 and 556).

\section{Statistical analysis}

We tested differences in descriptive statistics on demographic characteristics and baseline comorbidities between the exposed and non-exposed cohorts using chi-square 


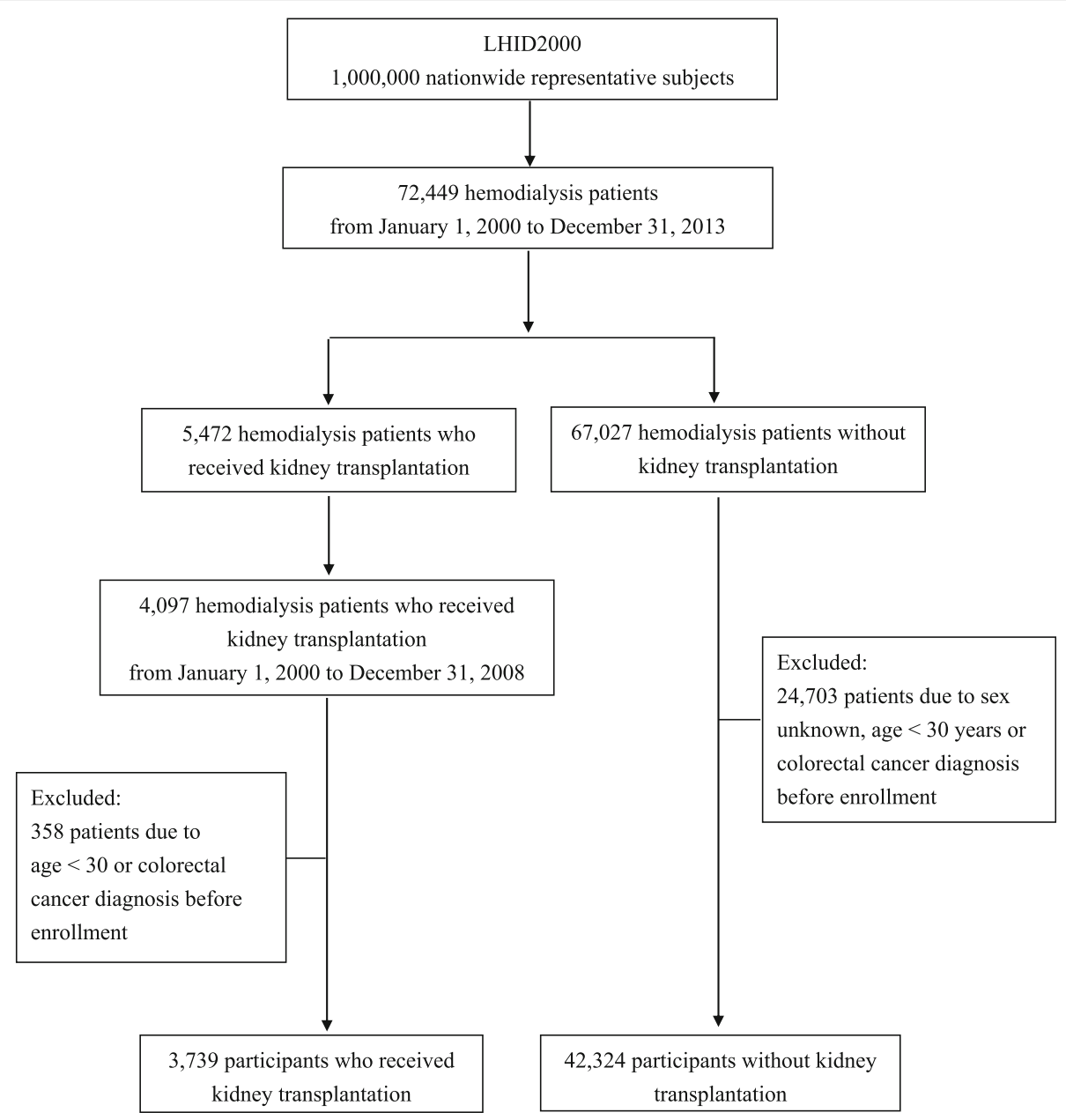

Fig. 1 Flow diagram of sample selection LHID Longitudinal Health Insurance Database

tests or Student's $t$-test when appropriate. In addition, we used Kaplan-Meier method to estimate the cumulative incidence of CRC. The log-rank test was performed to assess the difference in the cumulative incidence of CRC between the curves of the cohorts. In addition, we used Cox proportional hazards models to compute hazards ratios (HRs) with 95\% confidence intervals (CIs) to determine the association between $\mathrm{KT}$ and $\mathrm{CRC}$ risk after adjusting for the potential confounders. All statistical analyses were performed using SAS version 9.4 (SAS Institute Inc., Cary, NC), and we set the statistical significance at 0.05 for two-tailed tests.

\section{Ethics, consent and permission}

Since the dataset was released for research purposes and included only scrambled and anonymous information on patient identification, the study was exempt from informed consent from the subjects. Further, the execution of the present study and a waiver of obtaining informed consent from subjects have been approved by the Institutional
Review Board of Fu-Jen Catholic University (FJU-IRB NO: C104014).

\section{Results}

Table 1 summarizes the distribution of the baseline demographic characteristics and comorbidities of the cohorts. The mean age $( \pm \mathrm{SD})$ of the exposed and non-exposed cohorts was $61.84( \pm 12.63)$ and $52.31( \pm 14.74)$ years, respectively. The exposed cohort was markedly older than the non-exposed cohort. In addition, the exposed cohort had markedly higher proportions of males and comorbidities, including hypertension, hyperlipidemia, diabetes mellitus, coronary artery disease, congestive heart failure, stroke, asthma, and colorectal adenomas, than the non-exposed cohort. However, we observed no significant differences in terms of the proportions of obesity and colorectal polyps between the cohorts.

During the follow-up of 36,207 person-years, there were 135 CRC cases in the exposed cohort, resulting in an incidence rate of 372.9 per 100,000 person-years. On 
Table 1 Baseline demographics and comorbidities between Kidney transplantation and comparison cohorts

\begin{tabular}{|c|c|c|c|}
\hline Variable & $\begin{array}{l}\text { Kidney transplantation cohort } \\
N=3739(\%)\end{array}$ & $\begin{array}{l}\text { Comparison cohort } \\
N=42,324(\%)\end{array}$ & $p$-value \\
\hline Age, years (SD) & $61.84(12.63)$ & $52.31(14.74)$ & $<0.001$ \\
\hline Sex & & & $<0.001$ \\
\hline Female & $2207(59.03)$ & $23,258(54.95)$ & \\
\hline Male & $1532(40.97)$ & $19,066(45.05)$ & \\
\hline \multicolumn{4}{|l|}{ Comorbidity } \\
\hline Obesity & $38(1.02)$ & $467(1.10)$ & 0.624 \\
\hline Hypertension & $2994(80.07)$ & $18,245(43.11)$ & $<0.001$ \\
\hline Diabetes mellitus & $1537(41.11)$ & $9487(22.42)$ & $<0.001$ \\
\hline Hyperlipidemia & $1364(36.48)$ & $10,448(24.69)$ & $<0.001$ \\
\hline CAD & $1729(46.24)$ & 10,309 (24.36) & $<0.001$ \\
\hline Congestive heart failure & $616(16.47)$ & 2577 (6.09) & $<0.001$ \\
\hline Stroke & $694(18.56)$ & $3921(9.26)$ & $<0.001$ \\
\hline COPD & $1030(27.55)$ & $6230(14.72)$ & $<0.001$ \\
\hline Asthma & $648(17.33)$ & $4218(9.97)$ & $<0.001$ \\
\hline Colorectal polyps & $33(0.88)$ & $342(0.81)$ & 0.627 \\
\hline Colorectal Adenomas & $174(4.65)$ & $1611(3.81)$ & 0.010 \\
\hline
\end{tabular}

CAD Coronary artery disease, COPD Chronic obstructive pulmonary disease

the other hand, there were 889 CRC cases in the nonexposed cohort during the follow-up of 3,823,461 personyears, with an incidence rate of 232.5 CRC per 100,000 person-years. Figure 2 presents the Kaplan-Meier curves for the cumulative incidence of CRC for the two cohorts. The log-rank test revealed a significant difference in the cumulative risk of CRC between the cohorts over the entire Kaplan-Meier curve $(P<0.001)$. Notably, the differences were more robust with a prolonged duration of follow-up.

As shown in Table 2, the Cox proportional hazard regression model revealed that $\mathrm{KT}$ was significantly associated with an increased risk of CRC after adjusting for potential confounders (adjusted HR, 1.34; 95\% CI: 1.111.62). Globally, an increase in the risk of CRC related to $\mathrm{KT}$ has been observed in both sexes and all age groups. Notably, an elevated risk of CRC related to KT was more evident among women (adjusted HR, 1.48; 95\% CI: $1.15-1.91$ ) and those aged < 50 years (adjusted HR, 2.08; 95\% CI: 1.16-3.73).

\section{Discussion}

This nationwide, retrospective, population-based cohort study established that hemodialytic patients undergoing KT exhibit a markedly higher risk of CRC than those not undergoing KT. After adjusting for potential confounders, $\mathrm{KT}$ recipients had a 1.34-fold increased risk of CRC as compared with those not undergoing KT.

The introduction of potent immunosuppressive agents offered prolonged survival to transplant recipients, facilitating the documentation of an increased incidence of malignancies in this population [1-7, 18-21]. Thus, malignancies constitute a significant cause of late morbidity and mortality in renal allograft recipients. Compared with the general population, accumulating evidence suggests a higher risk of CRC in solid organ transplant recipients [18-23] and KT recipients [1-7]. The overall risk of CRC has increased by, at least, 2- to 2.5-fold in the kidney transplant population, and the pattern of elevated risk seems to be the highest in younger patients [1-7]. In a previous study, SIR for younger patients was 13.5, falling to $<3$ for older patients aged $\geq 55$ years compared with the age- and sex-matched general population [24]. Correspondingly, this study suggested that hemodialytic patients undergoing KT exhibit a markedly elevated risk of CRC compared with those not undergoing KT. In addition, the pattern of elevated risk was more pronounced among younger and female hemodialytic patients. Reportedly, immunosuppression following transplantation could be a critical factor in the amplified incidence of malignancy [6, 7]. Some studies have suggested that immunosuppression regimen biologically promotes systemic inflammation, resulting in immune dysregulation, increased DNA damage by upregulating the expression of tumor growth factor- $\beta$ and vascular endothelial growth factor, and altered gut microbiota, which are conducive to colorectal tumorigenesis [25, 26]. Conversely, a recent meta-analysis of cancer incidence including dialysis-dependent patients and KT recipients established no correlation between transplantation and CRC development [27]. These disparities 


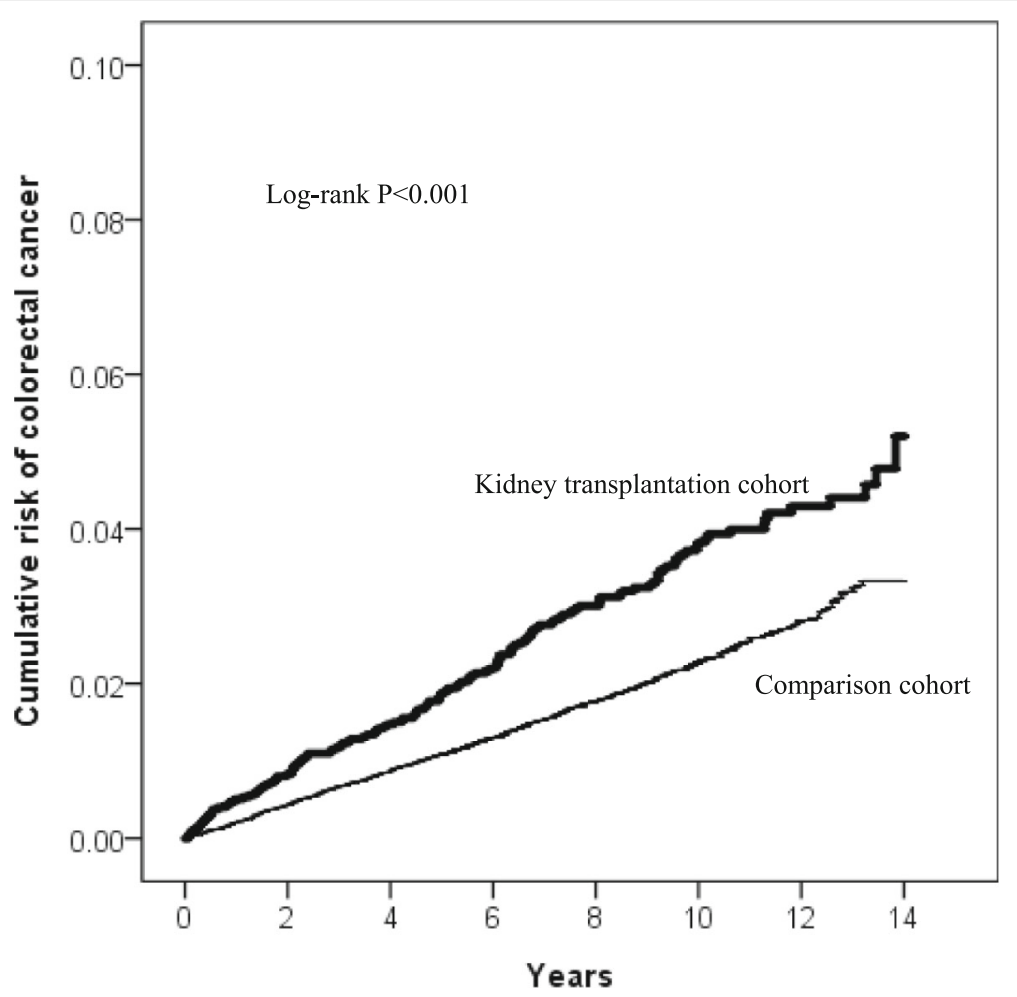

Fig. 2 Kaplan-Meier curves for the cumulative risk of colorectal cancer in kidney transplantation and comparison cohorts with the log-rank test

necessitate comprehensive studies to elucidate the risk of KT-associated CRC.

The findings of this study should be interpreted within the context of some limitations. Notably, studies based on insurance claims or other third-party data are often flawed because the information on confounding factors in insurance data is often limited [16, 28]. In this study, the information on major confounders, such as family history of CRC, obesity, smoking habits, and dietary patterns, was not available in the NHIRD. Thus, this study has residual confounding in the assessment of KTassociated CRC risk. Furthermore, the physical function before and after KT in hemodialytic patients was not assessed in the NHIRD.

Table 2 Multivariate associations between kidney transplantation and colorectal cancer (CRC) incidence among transplant recipients, overall and by age and sex

\begin{tabular}{|c|c|c|c|c|c|c|c|c|}
\hline \multirow[t]{2}{*}{ Variable } & \multicolumn{3}{|c|}{ Kidney transplantation cohort } & \multicolumn{3}{|c|}{ Comparison cohort } & \multirow[t]{2}{*}{$\mathrm{HR}(95 \% \mathrm{Cl})$} & \multirow{2}{*}{$\begin{array}{l}\text { Adjusted HR } \\
(95 \% \mathrm{Cl})\end{array}$} \\
\hline & No. CRC & PYs & Rate & No. CRC & PYs & Rate & & \\
\hline Overall & 135 & 36,207 & 372.9 & 889 & 382,346 & 232.5 & $1.59(1.33-1.91)$ & $1.34(1.11-1.62)$ \\
\hline \multicolumn{9}{|c|}{ Age group } \\
\hline$<50$ & 13 & 7225 & 179.9 & 198 & 187,138 & 105.8 & $1.64(0.93-2.90)$ & $2.08(1.16-3.73)$ \\
\hline $50-59$ & 27 & 8098 & 333.4 & 202 & 79,980 & 252.6 & $1.27(0.85-1.90)$ & $1.50(0.99-2.28)$ \\
\hline $60-69$ & 45 & 10,991 & 409.4 & 244 & 67,386 & 362.1 & $1.12(0.82-1.54)$ & $1.35(0.97-1.87)$ \\
\hline$\geqq 70$ & 50 & 9893 & 505.4 & 245 & 52,841 & 463.7 & $1.12(0.82-1.50)$ & $1.23(0.90-1.67)$ \\
\hline \multicolumn{9}{|c|}{ Sex } \\
\hline Female & 77 & 21,538 & 357.5 & 388 & 209,120 & 185.5 & $1.91(1.50-2.44)$ & $1.48(1.15-1.91)$ \\
\hline Male & 58 & 14,669 & 395.4 & 501 & 173,226 & 289.2 & $1.36(1.04-1.78)$ & $1.20(0.90-1.58)$ \\
\hline
\end{tabular}

$P Y_{S}$ Person-years, $H R$ Hazard ratio, $\mathrm{Cl}$ Confidence interval

Rate: incidence rate per 100,000 person-years

Hazard ratios were adjusted for age, sex, and comorbidities, including obesity, hypertension, diabetes, hyperlipidemia, coronary artery disease, congestive heart failure, stroke, chronic obstructive pulmonary disease, asthma, colorectal polyps, and colorectal adenomas 
Nevertheless, this study has some strengths. It is a national cohort study based on Taiwan's NHIRD, which contains data from Taiwan's compulsory and universal health care system that has a high coverage rate in Taiwan, facilitating analysis in a real-life setting of an unselected patient population. Furthermore, patient dropout was avoided, and selection bias minimized owing to the use of routine database records.

\section{Conclusions}

In conclusion, this study demonstrated that the CRC incidence in hemodialytic patients undergoing KT markedly increased compared with hemodialytic patients not undergoing KT. In addition, this study and biological plausibility support the association between KT and CRC. Although further research is warranted, the emerging data suggest that screening colonoscopy before and after transplantation will result in earlier detection of CRC, possibly decreasing mortality in this population. Indeed, according to the National Comprehensive Cancer Network (NCCN) guideline for colonoscopic negative/no polyps patients, the next colonoscopy should be recommended every 510 years [29]. However, routine CRC screening recommendations, such as colonoscopy, stool DNA tests or other stool based tests, may need to be revised after KT since individuals getting a transplant during the shorter follow-up period.

\section{Abbreviations \\ Cl: Confidence interval; CRC: Colorectal cancer; ESRD: End-stage renal disease; HR: Hazards ratio; ICD-9-CM: International Classification of Disease, Revision 9, Clinical Modification; KT: Kidney transplantation; LHID: Longitudinal Health Insurance Database; NCCN: National Comprehensive Cancer Network; NHI: National Health Insurance; NHIRD: National Health Insurance Research Database; NHRI: National Health Research Institute; RCIPD: Registry for Catastrophic Illness Patient Database; SIR: Standardized incidence ratio}

\section{Acknowledgements}

The authors thank the enrollees of the National Health Insurance Research Database.

for important contributions.

\section{Authors' contributions}

HEW contributed to protocol development, data analysis, and drafted the manuscript. YCL, JMH, and WCW contributed to conceived of the study and participated its coordination. WYC, YC Chen, YC Chou, and CFH contributed to data check and information retrieval. YFT and SLY researched the literature. CAS designed the study and contributed to overall management of the study. All authors reviewed the manuscript and approved the final version of the manuscript.

\section{Funding}

This study was supported by a grant from the Chi-Mei Medical Center (105CM-FJU-09). The funders had no role in the study design; collection, analysis, and interpretation of these data; writing the report; and the decision to submit the report for publication.

\section{Availability of data and materials}

With strict confidentiality guidelines being closely followed in accordance with personal electronic data protection regulations in Taiwan, the datasets used in the current study are not available from request.

\section{Ethics approval and consent to participate}

Since the dataset was released for research purposes and included only scrambled and anonymous information on patient identification, the study was exempt from informed consent from the subjects. Further, the execution of the present study and a waiver of obtaining informed consent from subjects have been approved by the Institutional Review Board of FuJen Catholic University (FJU-IRB NO:C104014).

Consent for publication

Not applicable.

\section{Competing interests}

The authors declare that they have no competing interests.

\section{Author details}

'Division of Nephrology, Department of Medicine, Tri-Service General Hospital, National Defense Medical Center, Taipei City, Taiwan. ${ }^{2}$ School of Public Health, National Defense Medical Center, Taipei City, Taiwan.

${ }^{3}$ Graduate Institute of Medical Sciences, National Defense Medical Center, Taipei City, Taiwan. ${ }^{4}$ Division of Colorectal Surgery, Department of Surgery, Tri-Service General Hospital, National Defense Medical Center, Taipei City, Taiwan. ${ }^{5}$ Department of Surgery, Suao and Yuanshan branches of Taipei Veterans General Hospital, Yilan County, Taiwan. ${ }^{6}$ Graduate Institute of Life Sciences, National Defense Medical Center, Taipei City, Taiwan. ${ }^{7}$ Department of Medicine, College of Medicine, Fu-Jen Catholic University, New Taipei City, Taiwan. ${ }^{8}$ Big Data Research Center, College of Medicine, Fu-Jen Catholic University, New Taipei City, Taiwan. ${ }^{9}$ Division of Colorectal Surgery, Department of Surgery, Chi-Mei Medical Center, Tainan City, Taiwan. ${ }^{10}$ Department of Health \& Nutrition, Chia Nan University of Pharmacy and Science, Tainan City, Taiwan. ${ }^{11}$ Department of Public Health, College of Medicine, Fu-Jen Catholic University, New Taipei City, Taiwan.

Received: 20 February 2019 Accepted: 23 October 2019

Published online: 16 November 2019

\section{References}

1. Birkeland S, Storm H. Cancer risk in patients on dialysis and after renal transplantation. Lancet. 2000;355(9218):1886-7.

2. Kyllönen L, Salmela K, Pukkala E. Cancer incidence in a kidney-transplanted population. Transpl Int. 2000;13(S1):S394-8.

3. Kasiske BL, Snyder JJ, Gilbertson DT, Wang C. Cancer after kidney transplantation in the United States. Am J Transplant. 2004;4(6):905-13.

4. Vajdic CM, McDonald SP, McCredie MR, van Leeuwen MT, Stewart JH, Law M, Chapman JR, Webster AC, Kaldor JM, Grulich AE. Cancer incidence before and after kidney transplantation. JAMA. 2006;296(23):2823-31.

5. Villeneuve P, Schaubel D, Fenton S, Shepherd F, Jiang Y, Mao Y. Cancer incidence among Canadian kidney transplant recipients. Am J Transplant. 2007;7(4):941-8.

6. Agraharkar ML, Cinclair RD, Kuo Y-F, Daller JA, Shahinian VB. Risk of malignancy with long-term immunosuppression in renal transplant recipients. Kidney Int. 2004;66(1):383-9.

7. Demir T, Ozel L, Gökçe A, Ata P, Kara M, Eriş C, Özdemir E, Titiz Mil: Cancer screening of renal transplant patients undergoing long-term immunosuppressive therapy. In: Transplantation Proceedings: 2015: Elsevier; 2015: 1413-1417.

8. Fitzmaurice C, Allen C, Barber RM, Barregard L, Bhutta ZA, Brenner H, Dicker DJ, Chimed-Orchir O, Dandona R, Dandona L. Global, regional, and national cancer incidence, mortality, years of life lost, years lived with disability, and disability-adjusted life-years for 32 cancer groups, 1990 to 2015: a systematic analysis for the global burden of disease study. JAMA Oncology. 2017;3(4): 524-48.

9. Grulich $\mathrm{AE}$, Van Leeuwen MT, Falster MO, Vajdic CM. Incidence of cancers in people with HIV/AIDS compared with immunosuppressed transplant recipients: a meta-analysis. Lancet. 2007;370(9581):59-67.

10. Wu HH, Kuo CF, Li IJ, Weng CH, Lee CC, Tu KH, Liu SH, Chen YC, Yang CW, Luo SF. Family aggregation and heritability of ESRD in Taiwan: a populationbased study. Am J Kidney Dis. 2017;70(5):619-26.

11. Chen C-J, You S-L, Lin L-H, Hsu W-L, Yang Y-W: Cancer epidemiology and control in Taiwan: a brief review. Japanese Journal of Clinical Oncology 2002, 32(suppl_1):S66-S81. 
12. Cancer registry annual report, 2013. [https://www.hpa.gov.tw/Pages/Detail. aspx? nodeid $=269 \&$ pid $=5191]$

13. Wu C-Y, Chen Y-J, Ho HJ, Hsu Y-C, Kuo KN, Wu M-S, Lin J-T. Association between nucleoside analogues and risk of hepatitis B virus-related hepatocellular carcinoma recurrence following liver resection. JAMA. 2012; 308(18):1906-13.

14. Chi C-C, Tung T-H, Wang J, Lin Y-S, Chen Y-F, Hsu T-K, Wang S-H. Risk of uveitis among people with psoriasis: a nationwide cohort study. JAMA Ophthalmology. 2017;135(5):415-22.

15. Cheng CL, Kao YHY, Lin SJ, Lee CH, Lai ML. Validation of the National Health Insurance Research Database with ischemic stroke cases in Taiwan. Pharmacoepidemiol Drug Saf. 2011;20(3):236-42.

16. Hsing AW, loannidis JP. Nationwide population science: lessons from the Taiwan national health insurance research database. JAMA Intern Med. 2015:175(9):1527-9.

17. Haggar FA, Boushey RP. Colorectal cancer epidemiology: incidence, mortality, survival, and risk factors. Clinics in Colon and Rectal Surgery. 2009; 22(04):191-7.

18. Collett D, Mumford L, Banner N, Neuberger J, Watson C. Comparison of the incidence of malignancy in recipients of different types of organ: a UK registry audit. Am J Transplant. 2010;10(8):1889-96.

19. Engels EA, Pfeiffer RM, Fraumeni JF, Kasiske BL, Israni AK, Snyder JJ, Wolfe RA, Goodrich NP, Bayakly AR, Clarke CA. Spectrum of cancer risk among US solid organ transplant recipients. JAMA. 2011:306(17):1891-901.

20. Adami J, Gäbel H, Lindelöf B, Ekström K, Rydh B, Glimelius B, Ekbom A, Adami H-O, Granath F. Cancer risk following organ transplantation: a nationwide cohort study in Sweden. Br J Cancer. 2003;89(7):1221.

21. Vajdic CM, van Leeuwen MT. Cancer incidence and risk factors after solid organ transplantation. Int J Cancer. 2009;125(8):1747-54.

22. Merchea A, Abdelsattar ZM, Taner T, Dean PG, Colibaseanu DT, Larson DW Dozois EJ. Outcomes of colorectal cancer arising in solid organ transplant recipients. Journal of Gastrointestinal Surgery : Official Journal of the Society for Surgery of the Alimentary Tract. 2014;18(3):599-604.

23. Merchea A, Shahjehan F, Croome KP, Cochuyt JJ, Li Z, Colibaseanu DT, Kasi PM. Colorectal Cancer characteristics and outcomes after solid organ transplantation. Journal of Oncology. 2019;2019:5796108.

24. Webster A, Craig J, Simpson J, Jones M, Chapman J. Identifying high risk groups and quantifying absolute risk of cancer after kidney transplantation: a cohort study of 15183 recipients. Am J Transplant. 2007:7(9):2140-51.

25. Herman M, Weinstein T, Korzets A, Chagnac A, Ori Y, Zevin D, Malachi T, Gafter U. Effect of cyclosporin a on DNA repair and cancer incidence in kidney transplant recipients. J Lab Clin Med. 2001;137(1):14-20.

26. Wilson ID, Nicholson JK. Gut microbiome interactions with drug metabolism, efficacy, and toxicity. Transl Res. 2017;179:204-22.

27. Shang W, Huang L, Li L, Li X, Zeng R, Ge S, Xu G. Cancer risk in patients receiving renal replacement therapy: a meta-analysis of cohort studies. Molecular and Clinical Oncology. 2016;5(3):315-25.

28. Hyman J. The limitations of using insurance data for research. J Am Dent Assoc. 2015;146(5):283-5.

29. Benson AB, Venook AP, Al-Hawary MM, Cederquist L, Chen YJ, Ciombor KK, Cohen S, Cooper HS, Deming D, Engstrom PF, et al. NCCN guidelines insights: Colon Cancer, version 2.2018. Journal of the National Comprehensive Cancer Network : JNCCN. 2018;16(4):359-69.

\section{Publisher's Note}

Springer Nature remains neutral with regard to jurisdictional claims in published maps and institutional affiliations.

Ready to submit your research? Choose BMC and benefit from:
- fast, convenient online submission
- thorough peer review by experienced researchers in your field
- rapid publication on acceptance
- support for research data, including large and complex data types
- gold Open Access which fosters wider collaboration and increased citations
- maximum visibility for your research: over 100M website views per year
At BMC, research is always in progress.
Learn more biomedcentral.com/submissions

